\title{
Reflectivity level of radio anechoic chambers
}

\section{Appel-Hansen, Jørgen}

\section{Published in:}

I E E E Transactions on Antennas and Propagation

Publication date:

1973

\section{Document Version}

Publisher's PDF, also known as Version of record

Link back to DTU Orbit

Citation (APA):

Appel-Hansen, J. (1973). Reflectivity level of radio anechoic chambers. I E E E Transactions on Antennas and Propagation, 21(4), 490-498.

\section{General rights}

Copyright and moral rights for the publications made accessible in the public portal are retained by the authors and/or other copyright owners and it is a condition of accessing publications that users recognise and abide by the legal requirements associated with these rights.

- Users may download and print one copy of any publication from the public portal for the purpose of private study or research.

- You may not further distribute the material or use it for any profit-making activity or commercial gain

- You may freely distribute the URL identifying the publication in the public portal

If you believe that this document breaches copyright please contact us providing details, and we will remove access to the work immediately and investigate your claim 
[38] K. Suetake, "Super broadband absorber," U.S. Patent 3623099 , Nov. 23, 1971

[39] R. E. Hiatt, E. F. Knott, and T. B. A. Senior, "A Study of VHF absorbers and anechoic rooms," Univ. Michigan, Ann Arbor, Rep. 5391-1-F.

[40] "Procedure of the free-space VSWR field probe technique," Emerson and Cuming, Inc., Canton, Mass., Tech. Bull.

[41] W. H. Emerson, "Anechoic chamber," U.S. Patent 3308 463, Mar. 7, 1967.

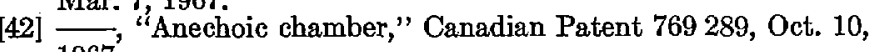
1967.

[43] $\frac{1967}{10}$ "Anechoic chamber," U.K. Patent 1062 142, Mar. 15, 1967.

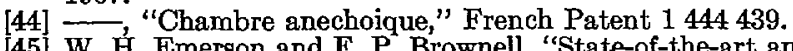

[45] W. H. Emerson and F. P. Brownell, "State-of-the-art anechoic chamber backscatter ranges," presented at M.I.T. Lincoln Lab. Radar Reflectivity Measurement Symp., June 1964.

[46] S. Galagan, "Understanding microwave absorbing materials and anechoic chambers," Microwaves, pt. I, Dec. 1969; pt. II, Jan. 1970; pt. III, Apr. 1970; pt. IV, May 1970.

[47] B. F. Goodrich Co. Reps. MR-07-MR-117, describing measurement of many anechoic chambers, 1960-1970.

[48] T. G. Hickman and T. J. Lyon, "Experimental evaluation of the Massachussetts Institute of Technology Lincoln Laboratory anechoic chamber," Scientific-Atlanta, Atlanta, Ga., Tech. Rep., 1968.

[49] J. Appel-Hansen, "Evaluation of a microwave anechoic chamber for one-way transmission," Lab. for Electromagnetic Theory, Tech. Univ. Denmark, Lyngby, Denmark, Rep R. 55, Jan. 1967
[50] H. Hollmann, "Antenna test chambers of the German Federal Postal Service," Nachrichtentech. Z., vol. 8, pp. 129-130, 1971.

[51] H. Uda, H. Komuro, and Y. Kunhora, "Wide band large microwave anechoic chamber," in 1969 Joint Conv. Rec. Electrical and Electronics Engineers of Japan.

[52] W. H. Emerson and H. B. Sefton, "An improved design for indoor ranges," Proc. IEEE, vol. 53, pp. 1079-1081, Aug. 1965.

[53] H. E. King, F. I. Shimabukuro, and J. L. Wong, "Characteristics of a tapered anechoic chamber," IEEE Trans. Antennas Propagat. (Commun.), vol. AP-15, pp. 488-490, May 1967.

[54] W. H. Emerson and F. P. Brownell, "Measurement of the horn shaped Bunker Ramo chamber," B. F. Goodrich Rep. MW-16, Aug. 2, 1964.

[55] W. H. Emerson, "Measurement of the B. F. Goodrich tapered chamber at the Marshall Space Flight Center," B. F. Goodrich Rep. MR-27, June 30, 1965.

[56] R. Garnham, "Solutions to Maxwell's equations in the tapered type of anechoic chamber," Royal Radar Establishment, Unpublished Memo.

[57] J. Steckel and W. Korvin, "Anechoic chamber looks up to cut costs," Microwaves, Sept. 1965.

[58] "Measurement of the NASA Goddard Space Flight Center vertical anechoic chamber," B. F. Goodrich Rep. MR-12, 1964.

[59] R. C. Johnson, "Antenna range for providing plane waves for antenna measurements," U.S. Patent 3302 205, Jan. 31, 1967.

[60] R. C. Johnson, H. Ecker, and M. R. Moore, "Compact range techniques and measurements," IEEE Trans. Antennas Propagat., vol. AP-17, pp. 568-576, Oct. 1969.

[61] $\mathrm{K}$. Kelleher, "Tests of microwave absorbing materials for tunnel antennas," Melpar Rep. 2, Contract AF19(6040)-1898.

\title{
Reflectivity Level of Radio Anechoic Chambers
}

\author{
J. APPEL-HANSEN
}

\begin{abstract}
A comparison between the antenna-pattern comparison technique and the free-space voltage standing-wave ratio technique for evaluating the reflectivity level of radio anechoic chambers is presented. Based on an analysis of the two techniques, it is pointed out which parameters influence the measured value of the reflectivity level. The comparison is illustrated with experimental results and it is explained why inconsistent and uncorrelated results may be found when the two methods are used. Furthermore, it is demonstrated, by introducing improvements in a chamber, how the reflectivity level can be used to measure the improvements. This work is inspired by the current discussion of finding a figure of merit for anechoic chambers. Based on the results, an evaluation procedure for anechoic chambers is indicated. However, it is pointed out and illustrated by examples that further investigations are necessary before a satisfactory procedure can be outlined.
\end{abstract}

\section{INTRODUCTION}

$\mathbf{D}$ UE TO THE progress in antenna and scattering studies, interest in indoor measurement ranges has increased since the first radio anechoic chambers were constructed in the early fifties. The advantage of radio

Manuscript received October 3, 1972; revised February 21, 1973. The author is with the Laboratory of Electromagnetic Theory, Technical University of Denmark, Lyngby, Denmark. anechoic chambers is, that measurements can be carried out under specific controlled conditions, for example, independent of weather conditions in nearly reflection-free regions and screened from disturbing signals.

Since the first chambers, there has been a current interest in studying methods by means of which it would be possible to characterize chambers by a figure of merit which indicates chamber performance or more precisely accuracy with which measurements can be carried out. It is generally accepted that for different types of measurements, it is necessary to use different methods to find a figure of merit. In this work a figure of merit characterizing radio anechoic chambers for antenna pattern measurements is discussed. The figure of merit is called the reflectivity level and indicates the inaccuracy in recorded pattern levels due to the small but inevitable reflections from the walls, floor and ceiling of the anechoic chamber lined with absorbing material.

It is well known that anechoic chambers may be constructed in many different ways and antennas possess very different radiation characteristics. Therefore, it is probably not possible to find only one value for the reflectivity level from which the measurement accuracy 
can be derived in all cases. Due to the complex problem several methods for obtaining the reflectivity level have been suggested. At this point it is illustrative to describe some previous works. Hiatt et al. [1] have given a survey of the most important evaluation methods used during the first ten years of anechoic chambers. Three of these methods were considered more satisfactory since the data which they provide can be analyzed to give numbers or curves to designate chamber performance. The development has since then shown that out of these three methods, two, usually referred to as the antenna-pattern comparison (APC) technique and the free-space voltage standing-wave ratio (VSWR) technique, are commonly accepted. It is often stated without experimental verification that the two techniques are essentially identical [1], [2]. Rethmeyer and Price [3] suggest using a combination of the two techniques. However, Kummer and Villeneuve [4] have compared the two techniques and found that inconsistent results were obtained. Hiatt et al. [1] have used the APC technique to evaluate the reflectivity level as a function of absorbing material lining a model of a chamber. They found that there was little correlation between the reflectivity levels obtained and the rating of absorbing material. In order to illuminate strongly all chamber surfaces, Clarke and Breithaupt [5] use detector mounts and coaxial-to-wave guide adapter flanges as antennas in a VSWR-technique evaluation of their chamber. In fact, since the results obtained for the reflectivity level depends very much on antenna gain, Hollmann [6], with the desire to be able to compare different anechoic chambers, suggests using probes with omnidirectional patterns as antennas.

This paper has three purposes. The first purpose is to give a comparison between the APC and the VSWR techniques. Before this comparison is given, the basic problem of finding the reflectivity level is discussed. Then the two techniques are described in detail. The possible reasons for the aforementioned inconsistency and uncorrelation is given. Furthermore, it is demonstrated experimentally that when properly applied, the two methods provide results which do not differ essentially. The present author has reported some of this comparison work earlier [7]. However, it was a laboratory report and some problems in applying the techniques were omitted. The second purpose is to demonstrate that the techniques may be used to evaluate improvements of radio anechoic chambers. The third purpose is to describe the state-of-art of evaluation of the reflectivity level.

It has to be mentioned that more interesting work concerning the dependence of the reflectivity level on the test parameters is to be carried out before a detailed knowledge of the informative value of the reflectivity level is obtained. However, it is hoped that persons reading this paper will get a broader background for estimating their measurement accuracy from given specifications of their chamber. Furthermore, persons who are going to evaluate their test range may be in a

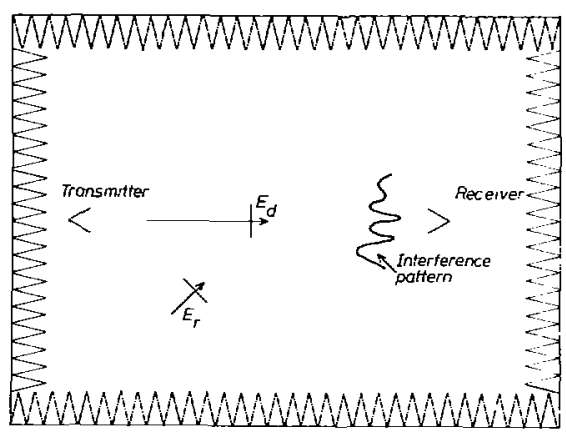

Fig. 1. General setup for recording patterns.

better position to dispose their test procedure depending upon their requirements. To help this, it is pointed out which parameters the measured value of the reflectivity level depends on. This is illustrated with some examples.

\section{Basic Considerations}

In order to make some basic considerations let us consider a general test set-up as shown in Fig. 1. A transmitting antenna is placed near one end wall in an anechoic chamber and the main lobe of the antenna is directed along the main axis of the chamber. A receiving antenna is placed on this axis at a proper distance from the transmitting antenna. Usually, the recciving antenna can be rotated in different manners and radiation patterns can be recorded. Furthermore, in order to carry out the experiments described in this paper, the support carrying the receiving antenna can be moved about.

When the transmitting antenna radiates, there exists at every point a signal which is composed of the direct signal $E_{d}$ from the transmitting antenna and a reflected signal $E_{r}$ from the surfaces of the chamber. The magnitude of the direct signal depends in the usual manner upon the distance from the transmitting antenna, whereas, the magnitude of the reflected signal varies in a complex manner with the position of the point under consideration. This complex variation is due to the fact that every point of the walls and other reflecting objects in the chamber reflects an incident signal. Furthermore, this incident signal is composed of a signal from the transmitting antenna and signals reflected from all other points of the walls of the anechoic chamber, and from, for example, the receiving antenna.

The reflected signal $E_{\mathrm{r}}$ will cause errors in measurements made in an anechoic chamber. A theoretical evaluation of the error is prevented due to the very complicated diffraction problem of finding the total interference pattern between $E_{r}$ and $E_{d}$ in the anechoic chamber. For determining the errors experimentally, much time is required, since measurement of the reflected field should be made at a sufficient number of points, so that it is possible to derive the destructive and constructive interference between $E_{d}$ and $E_{r}$ at every point in a specified zone of the chamber. This zone is the volume in which the actual measurements are made and is included in 


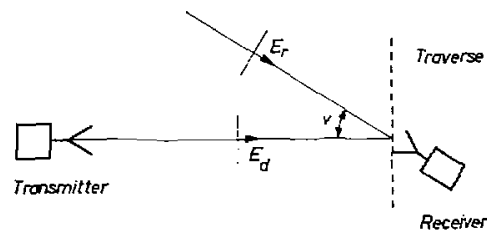

Fig. 2. Experimental arrangement for chamber evaluation.

the so-called quiet zone of the anechoic chamber. The quiet zone is the volume of the chamber in which certain specifications with respect to uniformity of the field are met. The extent of the quiet zone may for example be made dependent on how large a phase and amplitude difference of $E_{a}$ is specified between the center and the edge of the quiet zone. The "quietness" of this zone depends on the magnitude of reflections from the walls of the chamber.

As a measure of the reflected energy at a point in the quite zone, one may use the ratio of reflected power density to direct transmitted power density. This ratio will vary from point to point due to the complex variation of the reflected signal described in the preceding. To obtain a complete picture of the variation of the ratio requires much time. Furthermore, due to the fact that the reflected field in a chamber is the vector sum of many components arriving from different parts of the chamber, it is not possible to determine the magnitude of the reflected field with the general test setup for recording antenna patterns. This is seen from Fig. 2, where for simplicity we are assuming that we only have one reflected signal with amplitude $E_{r}$ and a direct signal with amplitude $E_{d}$. Furthermore, let the direction of propagation of the reflected signal be along the axis of the receiving antenna which makes an angle $v$ 'with respect to the transmitting antenma. As described in the following sections it is apparent that by moving the receiving antenna, the ratio between $E_{\mathrm{r}}$ and $E_{d}$ may be determined from the constructive and destructive interference recorded during the movement. However, because we never have only one reflected field, it is not the magnitude of the reflected field which is determined. This is evident from a consideration of $F i g .2$ in that case where $E_{r}$ is not propagating along the axis of the receiving antenna. What is really determined is an equivalent signal which, when propagating along the axis of the receiving antenna would cause a variation equal to that observed. This equivalent signal is often called the reflected signal.

The importance of finding the equivalent signal may be discussed. As seen in the preceding, it does not indicate the magnitude of the total reflected field. However, it indicates the magnitude of the error which may occur when a pattern is obtained. As it appears from the following sections, this is due to the fact that the equivalent signal is found from observing the limits within which the patterns are recorded.

It has to be noted that when we record antenna patterns for different positions of the receiving antenna, i.e., for different distances between the transmitting antenna and

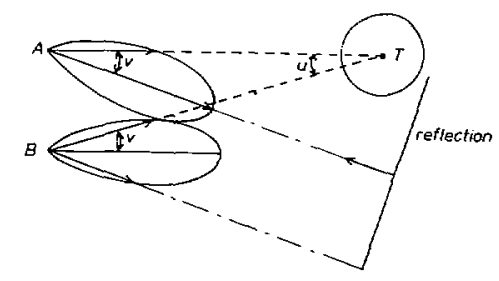

Fig. 3. APC technique. Detected incident energy constant.

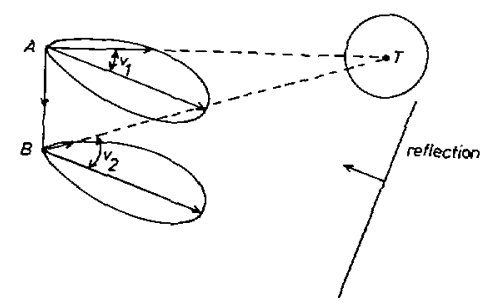

Fig. 4. VSWR technique. Detected reflected energy constant.

the receiving antenna, the variations in the recorded patterns are due to three effects. The first effect is the aforementioned constructive and destructive interference between the reflected signal and the direct signal. The second effect is variations in the amplitudes of the direct and the reflected signal. The third effect is variations in recorded patterns due to the fact that the far-field condition of illuminating the receiving antenna with a plane wave field can only be met to some extent depending upon the length of the test range. As it will be evident from the following sections, the first and second effect are taken into account in different manners depending upon the practising engineer. To the knowledge of the present author, the third effect is always neglected in evaluation of test ranges. In order to do this, the distance between the test antennas should be sufficiently large. The minimum distance may be determined by the usual far-field conditions which depend upon the antennas and the frequency. However, it cannot be disregarded that there may be cases where the determined reflectivity level is in part due to the third effect. This is owing to the fact that the far-field conditions only ensure that antenna patterns are recorded within a certain accuracy.

Owing to the difficulties in finding only one characteristic which describes the performance of the chamber, several test methods have been developed as mentioned in section I. It is characteristic for all the methods that the results depend upon the test procedures and the experimental arrangement which is supposed to be described by some test parameters. Before discussion of the dependence of the reflectivity level upon the test parameters and before setting up a scheme for test procedure, let us consider the two commonly used techniques, the APC- and VSWR techniques.

In order to illustrate the difference between the two techniques, let us consider Figs. 3 and 4. For simplicity, let us neglect variations in $E_{d}$ with distance, and suppose that the transmitter is an isotropic point source. Further, suppose that we have only one plane wave reflection, 
which has negligible effect on the main-lobe maximum, e.g., an effect less than $0.1 \mathrm{~dB}$. From Fig. 3 , it is seen that if we superimpose the patterns recorded at the shown positions $A$ and $B$, then at aspect angle $v$ the level of the detected reflected energy changes. On the other hand from Fig. 4, it is seen that if we try to keep the level of detected reflected energy constant during a continuous movement of the receiving antenna, then the level of the detected incident energy changes due to pattern scanning.

In both cases considered, it is tried to evaluate the influence of the reflection on pattern level. In fact, the two methods are the APC technique (Fig. 3) and the VSWR technique (Fig. 4). Before comparing the two techniques, it is convenient to describe them in detail and look at some results in the following sections.

\section{Antenna Patrern Comparison Technique}

\section{A. Theory}

When the pattern of the receiving antenna in the general test setup is recorded at, e.g., discrete positions on a traverse line in the quite zone, small variations occur in the patterns. From the magnitude of these variations the uncertainty of the recorded patterns is determined.

Let us again consider the situation in Fig. 2, where we only have one reflection. Let the pattern level (in $\mathrm{dB}$ ) at the angle $v$ be $a$ and let the detected field be $b$ and $c$ when the direct signal $E_{d}$ and the reflected signal $E_{r}$ are detected in-phase and out-of-phase, respectively. Then

$$
\begin{aligned}
b & =20 \log \frac{E_{d} \cdot 10^{a / 20}+E_{r}}{E_{d}} \\
c & =20 \log \frac{E_{d} \cdot 10^{\alpha / 20}-E_{r}}{E_{d}} \\
R & =20 \log \frac{E_{r}}{E_{d}}
\end{aligned}
$$

where $R$ is the ratio of reflected signal to direct signal in $\mathrm{dB}$. The parameter $R$ is called the reflectivity level. In order to obtain $b$ and $c$ all patterns are superimposed on one another so that their main-lobe peak levels coincide, see Fig. 5. From the values of $b$ and $c$ obtained in this manner, $R$ may be calculated from prepared curves or tables [7], [8]. From the basic considerations given in the preceding, it is understood that $R$ is not the ratio of reflected energy to direct energy in the test volume, but $R$ is related to this ratio in a complicated manner. Furthermore, since $R$ is a measure of pattern variations with receiving antenna position, $R$ may be used to indicate chamber performance.

It is evident from the preceding discussion that the value obtained for $R$ depends upon the level at which $R$ is found. Often the values of $R$ are averaged in different manners depending upon the antenna engineer using the APC technique. Since $R$ indicates the accuracy of patterns recorded in the chamber, the maximum value of $R$ which indicates maximum inaccuracy should also be reported.

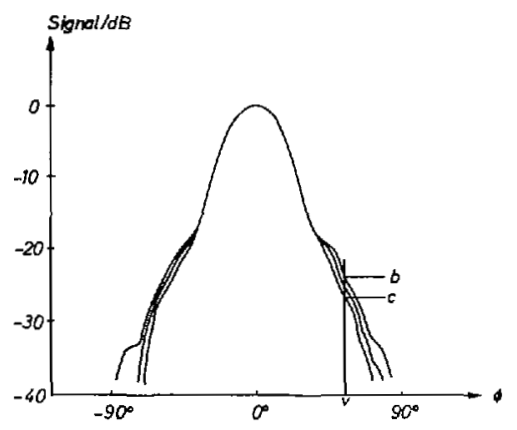

Fig. 5. Illustration of antenna pattern comparison test.

Besides the different manners in which $R$ is averaged, the superimposing of patterns is also carried out difforently. This is mainly due to the fact that during the measurements it may be decided to make one or both of the following two adjustments.

1) Usually, the setup is arranged such that $0^{\circ}$ on the chart paper correspond to the receiving antenna pointing in a direction parallel to the main axis of the chamber. By some means it may be possible to adjust the pattern recorded such that $0^{\circ}$ on the chart paper correspond to the receiving antenna pointing towards the transmitting antenna. In [3] is described the manner in which the adjustment is made when the receiving antenna is moved off the main axis of the chamber. The adjustment is carried out in order to avoid incorrect superimposing of the recorded patterns in the case where the reflections are so large that they change the direction of the main lobe.

2) During the measurements the main lobes of the different patterns are adjusted to the same level on the chart paper by changing the gain of the receiver. The purpose of the adjustment is to take into account variations in the direct signal due to scanning of the antenna patterns and distance variations between the antennas. It should be noted that in the case the reflections are so large that they influence the main-lobe level, the adjustment does not answer its purpose. Large reflections can be observed by moving the receiving antenna continuously along the main axis of the chamber while detecting the main-lobe level. If oscillations larger than a few tenths of a $\mathrm{dB}$ are observed, the reflections are large. In this case, the VSWR technique is recommended as a better technique for determining the reflectivity level.

The significance of making the aforementioned adjustments has not been investigated in the literature. It is evident that it depends on the experimental setup and the magnitude of the reflections, whether the adjustments influence the final result to a significant degree. In the following section, the usual procedure is described.

\section{B. Experimental Procedure}

The APC technique has been used to evaluate an anechoic chamber of dimensions $2 \times 4 \times 4 \mathrm{~m}^{3}$. Typical 16-dB standard gain antennas were used at $10 \mathrm{GHz}$. The transmitted field was vertically polarized and azimuth 
patterns were obtained. By moving the antenna along the main axis of the chamber while detecting the main-lobe level, it was observed that oscillations due to reflections were less than $0.2 \mathrm{~dB}$. Receiver gain adjustments were carried out so that the main-lobe level of the different patterns were recorded at the same level on the chart paper. In the analysis the recorded patterns were superimposed so that the main-lobes covered each other. The traverse line on which the patterns are recorded was a horizontal line normal to the line connecting the receiving and transmitting antennas. In total, 17 patterns were recorded at test points with an interspacing of a halfwavelength. One pattern was recorded on the axis of the room and 8 patterns were recorded on both sides of the axis. This means that the diameter of the zone examined was $24 \mathrm{~cm}$. Within this zone, the transmitting antenna sets up an essentially plane-wave field. In fact, the variation of the phase of the incident field was about $\lambda / 10$ when the distance between the antennas was $240 \mathrm{~cm}$. The APC technique was carried out for several traverse lines situated in the interval $2 \mathrm{~m}$ to $3 \mathrm{~m}$ from the transmitting antenna. From the 17 patterns recorded on the traverse line, the value of $R$ was found at the levels $-20,-25,-30$, and $-35 \mathrm{~dB}$. These levels corresponded to angles about $50^{\circ}, 60^{\circ}, 70^{\circ}$, and $75^{\circ}$. From the value of $R$ obtained, the maximum value was chosen to represent the maximum reflectivity in the interval $-90^{\circ}<\phi<90^{\circ}$ for $H$-plane pattern in the horizontal plane with 16-dB directive antennas. Some results are described in Section V.

\section{Free-Space VSWR Technique}

\section{A. Theory}

When performing the VSWR technique, the transmitting and receiving antennas are arranged as for the $\triangle P C$ technique. For a given aspect angle $\phi$ the receiving antenna is moved continuously on a traverse line. By coupling the linear motion of the receiving antenna to a recorder an interference pattern, often similar to a standing wave curve, is recorded as shown in Fig. 6. This curve shows the interference between the direct and reflected signals. It is seen that oscillations due to reflections are superimposed on the direct signal. Two types of variations are to be noticed. The amplitude of the oscillations varies due to the complicated variation of the reflected field with position. The average interference pattern level varies due to scanning of the two antenna patterns, distance variation between the antennas and variations in the far-field conditions. In the case of Fig. 6 changes in the direct signal are mainly due to scanning of the antenna patterns. This may easily be understood by considering Fig. 4, which was used to illustrate the artificial case where the detected reflected energy was kept constant.

The analysis of the standing wave curve is based on the following arguments. If a pattern is recorded, then the pattern level $a$ corresponding to the angle $\phi$ will be recorded with a tolerance determined by the maximum

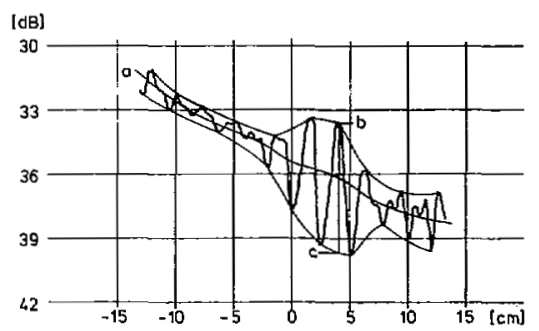

Fig. 6. Example of recorded standing wave curve at aspect angle $\phi=70^{\circ}$ at $10 \mathrm{GHz}$.

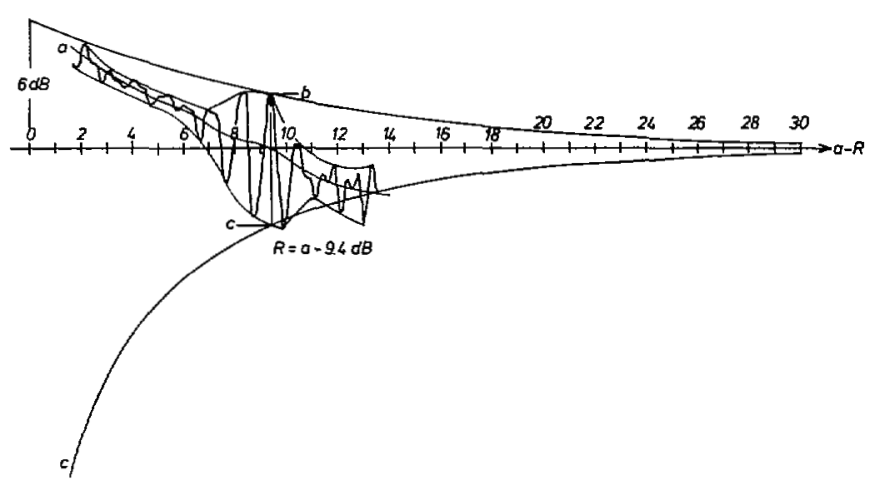

Fig. 7. Graphical determination of number of $\mathrm{dB}$, reflectivity level is below the pattern level.

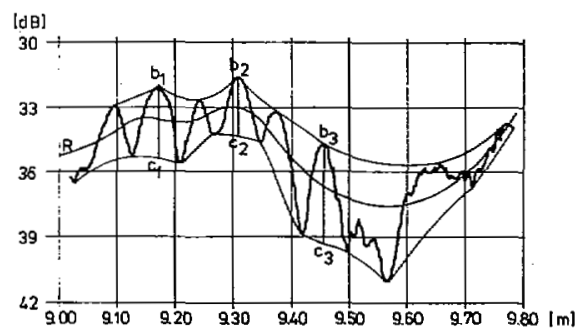

Fig. 8. Example of interference pattern when $a<R$ at $3 \mathrm{GHz}$.

VSWR recorded, see Fig. 6. As in the APC technique, the reflectivity level $R$ is determined from $b$ and $c$. In order to find $b$ and $c$ the standing wave is enveloped as shown in Fig. 6. Two curves, one connecting the maxima and another connecting the minima are loosely drawn. As illustrated, the value of $b$ and $c$ are chosen at that level where the maximum value of $R$ is obtained. From Fig. 6 it is seen that the maximum value of $R$ occurs at a pattern level of $a=-36.3 \mathrm{~dB}$. In Fig. 7 is shown the manner in which it is possible, graphically, to find the number of $\mathrm{dB}$ by which the reflectivity level is below the pattern level. Since $a$ is $-36.3 \mathrm{~dB}, R$ is found to be $-45.7 \mathrm{~dB}$. It is apparent that for the same value of $b-c$, the reflectivity level $R$ increases with the pattern level.

We have supposed that the detected reflected signal is smaller than the detected direct signal. That this is not always the case may be seen from Fig. 4 . It is apparent that due to pattern nulls, the direct signal may be smaller than the reflected signal for some positions and some aspect angles. In order to describe the manner in which the interference patterns are analysed in such case, it is illustrative to consider the case in Fig. 8. Here the am- 
plitude of the oscillations varies due to variations in the direct signal. The average level of the interference pattern varies due to the complicated variations of the reflected field. By analysing the interference pattern at its maximum level, the maximum value of $R$ is determined as the average interference pattern level. Before this analysis can be accomplished, it is necessary to identify the interference pattern as one for which the detected direct signal is smaller than the reflected signal. Of course this identification is easy, if the antenna pattern level is known beforehand. If this is not the case one may renounce information for the considered aspect angle. However, the ambiguity may be resolved, for example, by one or more of the following methods.

1) The interference pattern is analysed at say three different levels, see Fig. 8. From each analysis two numbers are found. Since the detected direct signal usually varies slowly in a regular way, it will often be possible to assign three of the obtained six numbers to the level of detected direct signal [9].

2) Preliminary numbers obtained for the detected pattern level and the reflectivity level are compared with numbers obtained for aspect angles close to the considered aspect angle. A continuity consideration in variation of $R$ and pattern level may solve the problem.

3) Symmetry in reflectivity level or pattern level may be used.

4) By introducing a metallic plate or by removing absorbers, the reflectivity level may be changed and the pattern level identified as an unchanged parameter.

It should be pointed out that the aforementioned methods may be used to identify pattern levels below the reflectivity level [9].

\section{B. Experimental Procedure}

The VSWR technique is made with the same experimental arrangement as used in the APC technique. The receiving antenna is moved along a horizontal line normal to the line connecting the receiving and transmitting antennas. The length traversed is $24 \mathrm{~cm}$. Since pattern levels $-20,-25,-30$, and $-35 \mathrm{~dB}$ correspond to aspect angles about $50^{\circ}, 60^{\circ}, 70^{\circ}$, and $75^{\circ}$, the standing waves are recorded at these angles. This is done in order to compare the results obtained by the VSWR technique with those obtained by the aforementioned APC technique. The results are described in Section $\mathrm{V}$.

\section{Comparison Betwewn Two Techniques}

\section{A. Theory}

After the description of the two techniques in details we are now able to continue the general remarks given earlier. The primary difference between the two techniques is that in performing the APC technique a continuous rotation is carried out at discrete positions and in the VSWR technique a continuous travel along a traverse is made for different aspect angles. In both methods the maximum value of reflectivity level $R$ may not be ob- served. In the antenna pattern technique, this can be solved by taking a large number of patterns. However, then the experiment time and analysis work is considerable. In the VSWR technique, $R$ may be determined for every $10^{\circ}$ and shown as a function of the aspect angle. At particular angles where $R$ seems to be maximum some additional interference patterns may be recorded and with a high degree of accuracy the largest value of $R$ may be determined graphically. The advantage of the APC technique is that in a test situation few patterns are easily recorded on top of each other on the same sheet of paper and in an illustrative manner. Thus, providing the antenna engineer with a preliminary feeling of the accuracy. However, if the engineer wishes to know the maximum discrepancy, it is recommended to apply the VSWR technique. Therefore, anechoic chamber specifications nowadays usually include the maximum values of the reflectivity level determined by the VSWR technique.

In order to show that at least in theory, the two techniques should give the same result, let us return to Figs. 3 and 4. It is seen from Fig. 3 which describes the APC technique, that the receiving antenna has the $0^{\circ}$ aspect angle when its axis is pointing towards the transmitting antenna. This is in accordance with the usual definition of the $0^{\circ}$ aspect angle. In Fig. 4 which describes the VSWR technique, the receiving antenna conveniently has the zero degree aspect angle when the antenna axis is pointing in directions parallel to the main axis of the chamber. This means, of course, due to the different definitions of aspect angle, we do not analyse the same interference patterns by using the APC and VSWR techniques as described in the previous sections. However, the reader can imagine the different manners in which this problem may be solved and the same interference patterns detected. This means that consistent results may be obtained by using the APC and VSWR techniques. This requires, however, many patterns in order to detect the worst case of interference from the largest reffection for a given angle of aspect. Furthermore, in order to analyze the interference pattern accurately it is required to extract a standing wave curve from the patterns. In fact, this is suggested in the first descriptions of the APC technique, and carried out by some users of this technique. However, patterns are usually only recorded at positions with an interspacing of one-half wavelength, which is often insufficient. Furthermore, when comparing with the VSWR technique the different definitions of aspect angle and the problems of superimposing of patterns are neglected. Moreover, the results obtained by the APC technique are averaged in different manners. This averaging, in fact, causes the largest difference between results obtained by the two techniques, since in the VSWR technique usually the largest value of $R$ is reported.

From the discussion made in this section and the previous ones, it is concluded that the APC technique conveniently gives a preliminary indication of the ac- 


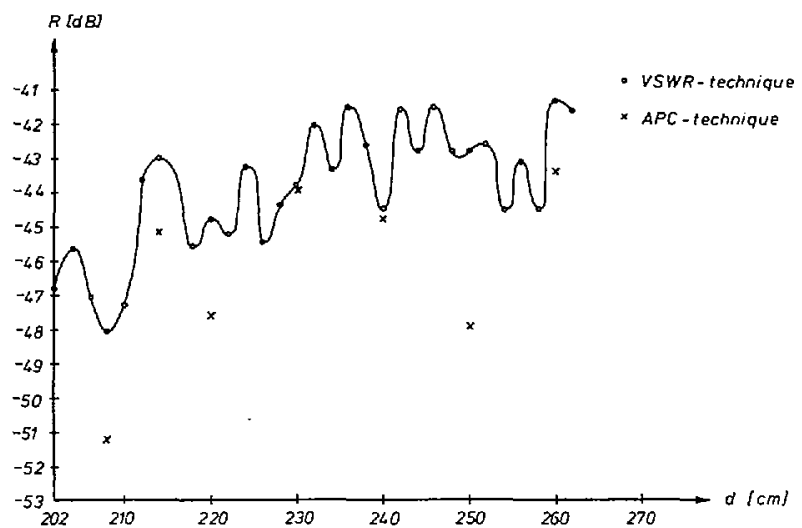

Fig. 9. Reflectivity level as function of distance $d$ between antennas.

curacy. However, the worst conditions may be missing and there are problems in interpreting the interference patterns. The VSWR technique finds the maximum reflectivity level with less time expenditure. Furthermore, the interference patterns are easily studied and reflections from specific parts of the anechoic chamber may be investigated in detail.

\section{B. Results}

In order to compare results obtained by the two techniques the aforementioned anechoic chamber is evaluated using the test procedures described in Sections III and IV. In Fig. 9 the maximum reflectivity level $R$ is shown as a function of the distance $d$ between the antennas. The distance varies from about $200 \mathrm{~cm}$ to $260 \mathrm{~cm}$.

The curve shown is obtained by the VSWR technique. It is seen that in accordance with the decrement of the incident field intensity with increasing spacing, the value of $R$ tends to increase. However, it is also noticed that small changes in the spacing may cause large changes in reflectivity level. Thus when the spacing is increased only $6 \mathrm{~cm}$ from $d=208 \mathrm{~cm}$, there is an increase of $5 \mathrm{~dB}$ in $R$.

The points marked with a cross are obtained by the APC technique. In performing this test the spacing is increased in steps of $10 \mathrm{~cm}$ from $d=220 \mathrm{~cm}$. Furthermore the APC technique is made at $d=208 \mathrm{~cm}$ and $214 \mathrm{~cm}$, where a relative minimum and maximum, respectively, is found by the VSWR technique. Comparing the results shown in Fig. 9, it is seen that the APC technique gives results which may indicate $5 \mathrm{~dB}$ better room performance than the results obtained by the VSWR technique. The discrepancy between the results is due to the differences between the two techniques.

The rapid increase in reflectivity level obseryed about $d=210 \mathrm{~cm}$ has been further investigated by recording the standing waves when the aspect angle is varied in steps of $5^{\circ}$ from $40^{\circ}$ to $90^{\circ}$. Thus the reflectivity level $R$ is determined as a function of aspect angle $\phi$ at the two positions $d=208 \mathrm{~cm}$ and $d=214 \mathrm{~cm}$. The analysis showed that there was a change of about $4 \mathrm{~dB}$ in the maximum values of $R$ in the interval $40^{\circ} \leq \phi \leq 90^{\circ}$. For angles smaller than $40^{\circ}, R$ is not determined since

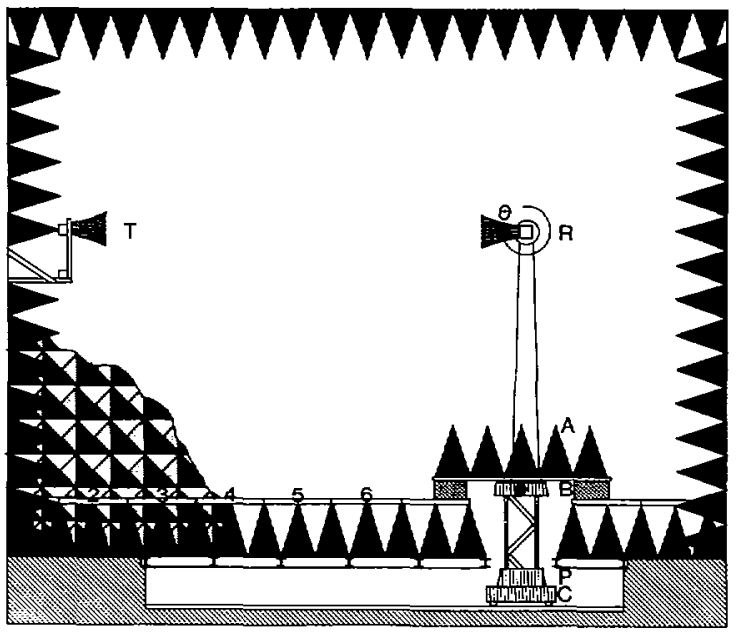

Fig. 10. Anechoic chamber.

here only very small changes (maximum $0.1 \mathrm{~dB}$ ) occur in the standing waves. This means that for $-40^{\circ} \leq \phi \leq$ $+40^{\circ}$ the pattern will be recorded very accurately.

\section{Reflectivity Level Used to INDICATE IMPROVEMENTS}

It is the purpose of this section to demonstrate that the reflectivity level obtained by the VSWR technique can be used to indicate the performance of radio anechoic chambers. This is done by measuring $R$ of the anechoic chamber shown in Fig. 10 under three different test conditions. In Fig. 10 is shown a longitudinal cross section of a $12 \times 14 \times 16 \mathrm{~m}^{3}$ rectangular chamber lined with 2-m high pyramidal absorbers. Inside the 463 floor absorbers are wooden poles which support 52 floor panels. The transmitting antenna $T$ is placed at one end of the chamber. The receiving antenna $R$ is mounted on a model tower which permits recording of three-dimensional antenna patterns. The model tower is by means of a tower base $B$ attached to an azimuth positioner $P$ placed on a cart $C$ which can run on rails in a pit. The reflectivity level is determined as a function of the angle $\theta$ in the $H$-plane of a horizontally polarized $20 \mathrm{~dB}$ standard gain horn. This means that $R$ is determined in the vertical plane where large reflections may occur when the receiving antenna is pointing towards the tower base $\left(\theta=270^{\circ}\right)$ or the specular reflection point $\left(\theta=310^{\circ}\right)$ of the floor. In order to screen reflections from the tower base and pit some absorbers $A$ are placed over the tower base. The reflection from the floor may be reduced by removing some floor panels. In the measurements $R$ was determined under three different measurement conditions: 1) floor panels in place and tower base absorbers removed; 2) floor panels and tower base absorbers in place; and 3 ) tower base absorbers in place and floor panels 2 to 6 in the middle of the chamber removed. In Fig. 11, the reflectivity level is shown as a function of aspect angle $\theta$ for the chamber in conditions 1) and 3). Under condition 1 ) it is scen that for $\theta=270^{\circ}$, there are large reflections due to the unscreened tower support mechanism. The maximum value is about $-37 \mathrm{~dB}$. It appears 


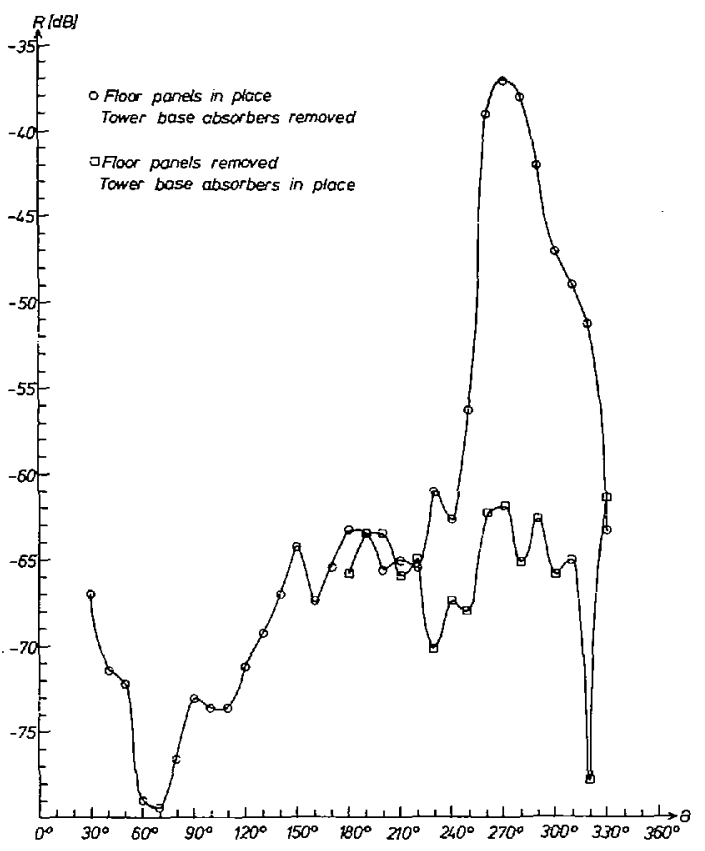

Fig. 11. Reflectivity level in vertical plane.

that when the tower base absorbers are in place and floor panels 2 to 6 removed the reflectivity level is below $-60 \mathrm{~dB}$. Thus, it is seen that when the chamber is improved from condition 1) to condition 3 ) there is a noticeable decrease in reflectivity level. Therefore, smaller improvements can also be detected. Thus, under condition 2), there was measured a decrease in reflectivity level of $18 \mathrm{~dB}$ for $\theta$ about $270^{\circ}$. However under this condition there is a maximum value of $R$ equal to $-48 \mathrm{~dB}$ at about $310^{\circ}$ due to specular reflections from the floor panels.

\section{Dependence of Reflectivity Level on Test Parameters}

As it may be apparent from the foregoing sections, the maximum value obtained for $R$ depends upon the following test parameters.

1) Frequency.

2) Directivity of transmitting and receiving antennas.

3) Polarization of the field.

4) Test plane in which $R$ is measured.

5) Traverse lines along which interference patterns are recorded.

6) Antenna support.

7) Positions of antennas in the chamber, in particular, distance from the internal surfaces of the chamber.

8) Number of interference patterns recorded.

9) Judicious interpretation of interference patterns.

10) Available measurement equipment.

To the knowledge of the author, detailed information about the dependence of the reflectivity level on all the test parameters does not seem to exist. May be this is due to the fact that the dependence of $R$ upon the test parameters is individual for the difference chambers. However, some dependences are known to some extent. It is usually stated that $R$ decreases with increasing
TABLE I

Reflectivity Level as Function of Directivity, Polartzation, and antenna Plane at $3 \mathrm{GHz}$

\begin{tabular}{|c|c|c|c|c|c|}
\hline \multirow[b]{2}{*}{$\begin{array}{l}\text { Direc- } \\
\text { tivity }\end{array}$} & \multirow[b]{2}{*}{$\begin{array}{c}\text { Polariza- } \\
\text { tion }\end{array}$} & \multirow{2}{*}{$\frac{\begin{array}{c}\text { Horizontal } \\
\text { Plane }\end{array}}{\text { Condi- }_{\text {tion 1) }}}$} & \multicolumn{3}{|c|}{ Vertical Longitudinal Plane } \\
\hline & & & $\begin{array}{l}\text { Condi- } \\
\text { tion } 1)^{\mathrm{a}}\end{array}$ & $\begin{array}{c}\text { Condi- } \\
\text { tion } 3)^{b}\end{array}$ & $\underset{\text { ment }}{\text { Improve- }}$ \\
\hline 20 & $\begin{array}{l}\text { Horizontal } \\
\text { Vertical } \\
\text { Horizontal } \\
\text { Vertical } \\
\text { Horizontal } \\
\text { Vertical }\end{array}$ & $\begin{array}{l}-23.1 \\
-22.0 \\
-45.6 \\
-37.8 \\
-54.4 \\
-45.9\end{array}$ & $\begin{array}{l}-22.0 \\
-24.0 \\
-36.2 \\
-36.0 \\
-37.2 \\
-40.5\end{array}$ & $\begin{array}{l}-32.0 \\
-29.2 \\
-52.4 \\
-42.8 \\
-61.8 \\
-54.8\end{array}$ & $\begin{array}{r}10.0 \\
5.2 \\
16.2 \\
6.8 \\
24.6 \\
14.3\end{array}$ \\
\hline
\end{tabular}

a Condition 1): Floor panels in place. Tower base absorbers removed.

b Condition 3): Floor panels removed. Tower base absorbers in place.

distance of the receiving antenna from the end wall. From Fig. 9 it is seen that this is correct to the extent that the measured value $R$ tends to decrease with increasing distance from the end wall, i.e., with decrease in $d$. However, the curve has large variations as discussed above. Other known dependencies are that $R$ tends to decrease with increase in frequency and directivity.

In order to illustrate the dependence of $R$ upon some of the test parameters, the chamber shown in Fig. 10 has been evaluated at $3 \mathrm{GHz}$ by using three types of antennas with a directivity of about 2,15 , and $20 \mathrm{~dB}$. The results are shown in Table I. The table indicates that the chamber is evaluated in the horizontal and longitudinal plane for horizontal and vertical polarization. The evaluation in the vertical plane is made under conditions 1) and 3) stated in the previous section. For each set of test parameters, $R$ is measured as a function of the aspect angle. The maximum value of $R$ for each set of the parameters is given in Table $\mathrm{I}$. This table also indicates the decrease which is observed in the maximum value of $R$ when the chamber is rearranged from condition 1) to condition 3), i.e., the chamber is improved.

Without going into detailed explanations which among others would involve consideration of antenna beam width in relation to chamber shape, the following effects should be observed from Table I.

1) The reflectivity level decreases with an increase in directivity. The average decrease is $16.4 \mathrm{~dB}$ and $7.3 \mathrm{~dB}$ when directivity is increased from $2 \mathrm{~dB}$ to $15 \mathrm{~dB}$ and from $15 \mathrm{~dB}$ to $20 \mathrm{~dB}$, respectively.

2) The reflectivity level depends upon polarization. For example in the case of $15 \mathrm{~dB}$ antennas, there is about $10 \mathrm{~dB}$ difference between the values of $R$ for vertical and horizontal polarization under condition 3 ).

3) The improvement is largest for high gain antennas. Thus, it is $24.6 \mathrm{~dB}$ for horizontally polarized $20-\mathrm{dB}$ antennas. For the low gain antennas it is only $10 \mathrm{~dB}$.

4) In the horizontal plane analysis has shown that reflections are largest from the back wall.

5) The reflectivity level is not the same in the vertical and horizontal plane under condition 1). The difference is largest for high gain antennas. 
6) For given directivity the maximum variations of $R$ with test parameters are $2,9.6$ and $17.2 \mathrm{~dB}$ for 2,15 and $20 \mathrm{~dB}$ antennas, respectively.

These results are obtained for the $12 \times 14 \times 16 \mathrm{~m}^{3}$ rectangular anechoic chamber mentioned above.

From the preceding it is understood that the test procedure which should be used to evaluate an anechoic chamber depends upon the requirements. A versatile chamber requires to be evaluated for several antennas with several polarizations and in several planes. Furthermore, the receiving antenna should be moved along several horizontal and vertical traverses, besides along several longitudinal lines. Basically, for a given directivity and frequency, there are $\mathbf{1 8}$ combinations of the three major planes, the three major transverse lines, and the two major polarizations. Thus a complete evaluation is very time-consuming.

In order to see whether a time reduction is possible, let us again consider the results given in Table I. It is seen that between the four different combinations for which the chamber is evaluated under condition 1), there is a spread of $2,9.6$, and $17.2 \mathrm{~dB}$ for antennas with 2,15 , and $20 \mathrm{~dB}$ directivity, respectively. From these results it is supposed that if the chamber is evaluated for a combination chosen arbitrarily from the 18 different possibilities then the obtained value of $R$ may deviate 10 to $20 \mathrm{~dB}$ from the worst case. However by a judicious selection of a combination from the 18 possibilities, it is assumed that the measured value of $R$ may deviate no more than 5 to $10 \mathrm{~dB}$ from the maximum value. Therefore, if this lack of accuracy in determining the largest value of $R$ can be accepted, it seems reasonable to evaluate the chamber for one proper combination of chamber plane, polarization and traverse line. This should be done for example at three frequencies by using three types of antennas, i.e., in total nine values of $R$ is determined. In the selection of test parameters, the following is to be taken into account: 1) frequency range of the chamber; 2) types of antennas which are to be measured in the chamber; and 3) those parts of the internal surfaces of the chamber from which the largest reflections are to be expected.

Finally, since improvements are measured more effectively with high gain antennas than with low gain antennas, comparison of chambers are easier made from results obtained with high gain antennas than results obtained with low gain antennas. However, low gain antennas should also be used because they indicate the largest values of the reflectivity level.

\section{Conclusion}

The state-of-the art of evaluation of the reflectivity level in radio anechoic chambers has been described. A comparison between the APC-technique and the VSWR technique has shown that the APC technique gives results which generally indicate a better room performance than the results obtained by VSWR technique. This is mainly due to the fact that in the APC technique the patterns are recorded at discrete steps. However, if a considerable number of patterns are recorded and properly analyzed the two techniques give the same results. This, however, requires a high expenditure of time. From the comparison it is concluded that in an actual measurement situation, the APC technique conveniently gives a preliminary indication of measurement accuracy, but the VSWR technique is the more accurate method for measuring the reflectivity level in an anechoic chamber.

It has been found that the reflectivity level can be used to indicate improvements in radio anechoic chambers. This is done by determining the reflectivity level before and after screening of the antenna support mechanism and removal of floor panels during test in an anechoic chamber. The maximum value of the reflectivity level was improved from $-37 \mathrm{~dB}$ to $-62 \mathrm{~dB}$ in the case of antennas with a gain of $20 \mathrm{~dB}$. Improvements are not so noticeable by means of low-gain antennas as by means of high-gain antennas.

The dependence of the reflectivity level on such test parameters as pattern plane, antenna gain and polarization is demonstrated by examples. Thus it is shown that for one antenna there is a variation of 10 to $20 \mathrm{~dB}$ in $R$ as a function of test parameters. The variation is largest for high gain antennas. However, it is pointed out that more knowledge concerning the dependence of reflectivity level on test parameters is desirable before satisfactory evaluation procedures for anechoic chambers can be outlined. Therefore, if the measurement accuracy must be known accurately, it is necessary to evaluate the chamber by the VSWR technique for the actual measurement situation.

\section{ACKNOWLEDGMENT}

The author is grateful to N. O. Nielsen and P. Laugesen for their assistance during the measurements and analysis of the data. The author thanks K. Cronberg-Ipsen for reading and commenting on the manuscript.

\section{REFERENCES}

[1] R. E. Hiatt, E. F. Knott, and T. B. A. Senior, "A study of VHF absorbers and anechoic rooms," Radiation Lab., Univ. Michigan, Tech. Rep. 5391-1-F, Feb. 1963.

[2] R. R. Bowman, "Prevalent methods for evaluating anechoic chambers: some basic limitations," presented at 1966 Measurement Seminar, Session III, Lecture 3 of High Frequency and Microwave Field Strength Precision NBS Rep. 9229.

[3] B. D. Rethmeyer and W. A. Price, "Procurement and evaluation of anechoic chamber," U. S. Naval Avionic Facility, Indianapolis, Ind., Tech. Rep. 403, July 1964.

[4] W. H. Kummer and A. T. Villeneuve "Experimental evaluation of anechoic chambers," in 1965 IEEE G-AP Int. Symp. Prog. and Dig., pp. 301-306.

[5] B. Clarke and R. Breithaupt, "Test measurements on a $13 \mathrm{ft}$. $X$ $9.8 \mathrm{ft}$. $\times 7.5 \mathrm{ft}$. microwave anechoic chamber," Communication Res. Cent., Nat. Space Telecommun. Lab., CRC Tech. Note 633, Aug. 1971.

[6] H. Hollman, "Design and function of anechoic funnel and rectangular chambers for antenna tests," Fernmeldetech. $Z$. der Deutschen Bundespost, A 454 TBr 13, Nov. 1971.

[7] J. Appel-Hansen, "Evaluation of a microwave anechoic chamber for one-way transmission," Tech. Univ. Denmark, Lyngby, Denmark, R 55, Jan. 1967 .

[8] E. F. Buckley, "Design, evaluation and performance of modern microwave anechoic chambers for antenna measurements," Electron. Combonents, pp. 1119-1126, Dec. 1965.

[9] J. Appel-Hansen, "Experimental methods for accurate determination of low antenna pattern levels in microwave anechoic chambers," Radio Anechoic Chamber, Tech. Univ. Denmark, Lyngby, Denmark, R 57, Apr. 1967. 